\title{
Non-deterministic Connectives in Propositional Gödel Logic
}

\author{
Ori Lahav Arnon Avron \\ School of Computer Science, Tel Aviv University
}

\begin{abstract}
We define the notion of a canonical Gödel system in the framework of single-conclusion hypersequent calculi. A corresponding general (nondeterministic) Gödel valuation semantics is developed, as well as a (non-deterministic) linear intuitionistic Kripke-frames semantics. We show that every canonical Gödel system induces a class of Gödel valuations (and of Kripke frames) for which it is strongly sound and complete. The semantics is used to identify the canonical systems that enjoy (strong) cut-admissibility, and to provide a decision procedure for these systems. The results of this paper characterize, both proof-theoretically and semantically, a large family of (non-deterministic) connectives that can be added to propositional Gödel logic.
\end{abstract}

Keywords: Propositional Gödel Logic, Nondeterministic Semantics, Hypersequent Calculi

\section{Introduction}

Fuzzy logics is a vast field of research with numerous applications. The main idea behind the development of fuzzy logic is that in many cases propositions do not have a crisp truth value. Fuzzy logics usually try to solve this problem by allowing the whole range of numbers between 0 and 1 to serve as potential "truth values" for propositions. By this they strongly deviate from classical logic, which employs just the two extreme values: 0 and 1. However, current fuzzy logics are still orthodox (deterministic) many-valued logics, and all of them are based on the principle of truth-functionality: the truth-value of a compound formula is uniquely determined by the truth-values of its subformulas. Thus in the standard logical formalization of Zadeh's theory (see Hájek's monograph [6]) the conjunction and implication connectives are respectively interpreted by some (left-)continuous t-norm on the interval $[0,1]$ and its residuum. Accordingly, the phenomenon of fuzziness is limited in these theories to atomic formulas, but no fuzziness is allowed in the semantics of connectives: an interpretation of an $n$-ary connective is always a crisp $n$-ary function on the interval $[0,1]$. This fact leads to many counter-intuitive results. Thus if two formulas get by chance the same truth value $(0.7$, say), then they will be treated as absolutely equivalent, even if there is little connection between them (e.g. one says that John is tall, the other says that Mary is young). Moreover: given any two formulas $\varphi$ and $\psi$, either $\varphi \rightarrow \psi$ or $\psi \rightarrow \varphi$ is absolutely true (i.e. it is assigned the classical truth-value 1), no matter how relevant one is to the other.

One possible direction for solving this problem is to relax the truth-functionality principle. This led to the introduction (in [3]) of non-deterministic matrices (Nmatrices) - a natural generalization of ordinary multi-valued matrices, in which the truthvalue of a complex formula can be chosen nondeterministically out of some non-empty set of options.

In this paper we provide a first step towards a theory of non-deterministic semantics for fuzzy logics. We choose the framework of Gödel logic, and characterize a large set of non-deterministic connectives that can be added to the usual connectives used in Gödel logic. Gödel logic is probably the most important intermediate logic (i.e. a logic between intuitionistic logic and classical logic), which turns up in several places. Recently it has again attracted a lot of attention because of its recognition as one of the three most basic fuzzy logics [6].

A natural question now is how exactly a new connective is defined? While there is more than one way to address this issue, we follow the prooftheoretic approach, according to which the meaning of a connective is determined by the introduction and elimination rules which are associated with it (see e.g. [8] for discussions and references). Here one usually has in mind a deduction system of an ideal type, where each connective has its own introduction and elimination rules, and these rules should meet the following conditions: in a rule for some connective this connective should be mentioned exactly once, and no other connective should be involved. This approach was applied in [3] to characterize the set of semi-classical connectives, and in [4] to characterize the set of basic constructive connectives. However, these papers deal with (single-conclusion or multiple-conclusion) sequent system, while the most useful proof-theoretical framework for fuzzy logics is that of hypersequent systems (see [5] and [7]). In particular, the only known system for Gödel logic, which is of the 'ideal' type described above, is the hypersequential system HG (introduced in [1]), which has exactly the same logical rules as the usual 
Gentzen-type system for propositional intuitionistic logic. Thus we use the framework of hypersequential calculi to provide a proof-theoretic characterization of (deterministic and non-deterministic) connectives in Gödel logic. This characterization is accompanied (in sections 4 and 5) by semantic characterizations. Two kinds of semantic characterizations are provided: a many-valued semantics - by generalizing Gödel valuations, and a Kripkestyle semantics - by generalizing linear intuitionistic Kripke-frames. The latter is also used to prove a strong cut-admissibility theorem for the family of hypersequent systems we introduce, and to provide a decision procedure for all the logics induced by the systems of this family. The equivalence between the two types of semantics is established in Section 6 .

\section{Preliminaries}

In what follows $\mathcal{L}$ is a propositional language, and $\operatorname{Frm}_{\mathcal{L}}$ is its set of wffs. We assume that the atomic formulas of $\mathcal{L}$ are $p_{1}, p_{2}, \ldots$.

Definition 1. A sequent is an expression of the form $\Gamma \Rightarrow E$ where $\Gamma$ and $E$ are finite sets of formulas, and $E$ is either a singleton or empty. A sequent of the form $\Gamma \Rightarrow\{\varphi\}$ is called definite. A sequent of the form $\Gamma \Rightarrow \emptyset$ is called negative. A clause is a sequent which consists of atomic formulas only.

Definition 2. A hypersequent is a finite set of sequents.

We shall use the usual hypersequent notation $s_{1}$ | $\ldots \mid s_{n}$ instead of $\left\{s_{1}, \ldots, s_{n}\right\}$. We also employ the standard abbreviations, e.g. $\Gamma, \psi \Rightarrow E$ instead of $\Gamma \cup\{\psi\} \Rightarrow E$, and $H \mid s$ instead of $H \cup\{s\}$.

Given a set $\mathcal{H}$ of hypersequents, we denote by $\operatorname{frm}[\mathcal{H}]$ the set of formulas that appear in $\mathcal{H}$.

Definition 3. An $\mathcal{L}$-substitution is a function $\sigma: \operatorname{Frm}_{\mathcal{L}} \rightarrow \operatorname{Frm}_{\mathcal{L}}$, such that for every $n$-ary connective $\diamond$ of $\mathcal{L}$, we have: $\sigma\left(\diamond\left(\psi_{1}, \ldots, \psi_{n}\right)\right)=$ $\diamond\left(\sigma\left(\psi_{1}\right), \ldots, \sigma\left(\psi_{n}\right)\right)$. A substitution is extended to sets of formulas in the obvious way: $\sigma(\mathcal{T})=$ $\{\sigma(\varphi) \mid \varphi \in \mathcal{T}\}$ (in particular, $\sigma(\emptyset)=\emptyset$ ).

Given formulas $\psi_{1}, \ldots, \psi_{n}, \sigma_{\psi_{1}, \ldots, \psi_{n}}$ denotes the substitution $\sigma$ such that $\sigma\left(p_{i}\right)=\psi_{i}$ for $1 \leq i \leq n$ and $\sigma\left(p_{i}\right)=p_{i}$ for $i>n$.

\section{Canonical Gödel Systems}

The following definitions formulate in exact terms the structure of hypersequent rules that are used to characterize (non-deterministic) connectives in Gödel logic. These definitions are the hypersequent versions of Definitions 3.1 and 3.2 in [4]. The reader is referred to [4] for more details.

\section{Definition 4.}

1. A single-conclusion canonical right introduction rule for a connective $\diamond$ of arity $n$ is an expression of the form: $\mathcal{S} / \Rightarrow \diamond\left(p_{1}, \ldots, p_{n}\right)$, where $\mathcal{S}$ is a finite set of clauses over $\left\{p_{1}, \ldots, p_{n}\right\}$.

2. A (hypersequential) application of the rule $\left\{\Pi_{i} \Rightarrow E_{i}\right\}_{1 \leq i \leq m} / \Rightarrow \diamond\left(p_{1}, \ldots, p_{n}\right)$ is any inference step inferring $H \mid \Gamma \Rightarrow \sigma\left(\diamond\left(p_{1}, \ldots, p_{n}\right)\right)$ from the set $\left\{H \mid \Gamma, \sigma\left(\Pi_{i}\right) \Rightarrow \sigma\left(E_{i}\right)\right\}_{1 \leq i \leq m}$, where $H$ is an arbitrary hypersequent, $\Gamma$ is a finite set of formulas, and $\sigma$ is an $\mathcal{L}$-substitution.

3. A single-conclusion canonical left introduction rule for a connective $\diamond$ of arity $n$ is an expression of the form: $\mathcal{S}_{1}, \mathcal{S}_{2} / \diamond\left(p_{1}, \ldots, p_{n}\right) \Rightarrow$, where $\mathcal{S}_{1}$ is a finite set of clauses over $\left\{p_{1}, \ldots, p_{n}\right\}$, and $\mathcal{S}_{2}$ is a finite set of negative clauses over $\left\{p_{1}, \ldots, p_{n}\right\}$.

4. A (hypersequential) application of the rule $\left\{\Pi_{i} \Rightarrow E_{i}\right\}_{1 \leq i \leq m},\left\{\Sigma_{i} \Rightarrow\right\}_{1 \leq i \leq k} / \diamond\left(p_{1}, \ldots, p_{n}\right) \Rightarrow$ is any inference step inferring $H \mid \Gamma, \sigma\left(\diamond\left(p_{1}, \ldots, p_{n}\right)\right) \Rightarrow E \quad$ from the sets $\quad\left\{H \mid \Gamma, \sigma\left(\Pi_{i}\right) \Rightarrow \sigma\left(E_{i}\right)\right\}_{1 \leq i \leq m} \quad$ and $\left\{H \mid \Gamma, \sigma\left(\Sigma_{i}\right) \Rightarrow E\right\}_{1 \leq i \leq k}$, where $H$ is an arbitrary hypersequent, $\Gamma \Rightarrow E$ is an arbitrary sequent, and $\sigma$ is an $\mathcal{L}$-substitution.

Convention: From now on, by "right (left) rule" we shall mean "single-conclusion canonical right (left) introduction rule".

Example 5. The two usual rules for implication are:

$$
\begin{gathered}
(\Longrightarrow): \quad\left\{p_{1} \Rightarrow p_{2}\right\} / \Rightarrow p_{1} \supset p_{2} \\
(\supset \Rightarrow): \quad\left\{\Rightarrow p_{1}\right\},\left\{p_{2} \Rightarrow\right\} / p_{1} \supset p_{2} \Rightarrow
\end{gathered}
$$

Applications of these rules have the form:

$$
\frac{H \mid \Gamma, \varphi \Rightarrow \psi}{H \mid \Gamma \Rightarrow \varphi \supset \psi} \quad \frac{H|\Gamma \Rightarrow \varphi \quad H| \Gamma, \psi \Rightarrow \theta}{H \mid \Gamma, \varphi \supset \psi \Rightarrow \theta}
$$

Example 6. Suppose we introduce a "semiimplication" $\leadsto$ with the following two rules:

$$
\begin{gathered}
(\Longrightarrow): \quad\left\{p_{2}\right\} / \Rightarrow p_{1} \leadsto p_{2} \\
(\leadsto): \quad\left\{\Rightarrow p_{1}\right\},\left\{p_{2} \Rightarrow\right\} / p_{1} \leadsto p_{2} \Rightarrow
\end{gathered}
$$

Applications of these rules have the form:

$$
\frac{H \mid \Gamma \Rightarrow \psi}{H \mid \Gamma \Rightarrow \varphi \leadsto \psi} \quad \frac{H \mid \Gamma \Rightarrow \varphi \Gamma, \psi \Rightarrow \theta}{H \mid \Gamma, \varphi \sim \psi \Rightarrow \theta}
$$

Example 7. Suppose we introduce an "asterisk connective" * with the following two rules:

$$
\begin{array}{cc}
(\Rightarrow *): & \left\{\Rightarrow p_{1}, \Rightarrow p_{2}\right\} / \Rightarrow p_{1} * p_{2} \\
(* \Rightarrow): & \emptyset,\left\{p_{1} \Rightarrow, p_{2} \Rightarrow\right\} / p_{1} * p_{2} \Rightarrow
\end{array}
$$

Applications of these rules have the form:

$$
\begin{gathered}
\frac{H|\Gamma \Rightarrow \varphi \quad H| \Gamma \Rightarrow \psi}{H \mid \Gamma \Rightarrow \varphi * \psi} \\
\frac{H|\Gamma, \varphi \Rightarrow \theta \quad H| \Gamma, \psi \Rightarrow \theta}{H \mid \Gamma, \varphi * \psi \Rightarrow \theta}
\end{gathered}
$$


Definition 8. A canonical Gödel system is a (single-conclusion) hypersequential calculus whose axioms are the hypersequents of the form $\varphi \Rightarrow \varphi$; cut, internal weakening, external weakening, and the communication rule are among its rules; and each of its other rules is a single-conclusion canonical rule.

Remark 9. The cut rule allows the following derivations:

$$
\frac{H|\Gamma \Rightarrow \varphi \quad H| \Gamma, \varphi \Rightarrow E}{H \mid \Gamma \Rightarrow E}
$$

The internal and external weakening rules allow the following derivations:

$$
\frac{H \mid \Gamma \Rightarrow E}{H \mid \Gamma, \Delta \Rightarrow E} \quad \frac{H \mid \Gamma \Rightarrow}{H \mid \Gamma \Rightarrow \psi} \quad \frac{H}{H \mid \Gamma \Rightarrow E}
$$

The communication rule allows the following derivations:

$$
\frac{H\left|\Gamma, \Delta \Rightarrow E_{1} \quad H\right| \Gamma, \Delta \Rightarrow E_{2}}{H\left|\Gamma \Rightarrow E_{1}\right| \Delta \Rightarrow E_{2}}
$$

We denote by $\vdash_{\mathbf{G}}$ the syntactic consequence relation between hypersequents induced by a canonical Gödel system $\mathbf{G}$ (i.e. $\mathcal{H} \vdash_{\mathbf{G}} H$ iff there exists a derivation in $\mathbf{G}$ of the hypersequent $H$ from the set of hypersequents $\mathcal{H}$ ).

Definition 10. Let $\mathbf{G}$ be a canonical Gödel system, $\mathcal{H} \cup\{H\}$ be a set of hypersequents, and $\mathcal{F}$ be a set of formulas.

1. A proof in $\mathbf{G}$ of $H$ from $\mathcal{H}$ is called a $\mathcal{F}$-proof if the cut-formula of every cut in the proof is a formula from $\mathcal{F}$.

2. $\mathcal{H} \vdash_{\mathbf{G}}^{\mathcal{F}} H$ if there exists an $\mathcal{F}$-proof in $\mathbf{G}$ of $H$ from $\mathcal{H}$.

We say that $\mathbf{G}$ enjoys strong cut-admissibility if $\mathcal{H} \vdash_{\mathbf{G}} H$ implies $\mathcal{H} \vdash_{\mathbf{G}}^{\text {frm }[\mathcal{H}]} H$ for every set $\mathcal{H} \cup\{H\}$ of hypersequents.

The property of strong cut-admissibility is crucial to ensure that a set of rules for a connective can be seen as a definition of the connective (see [4]). In the sequel we prove that the following condition of coherence (defined exactly as in [4]) characterizes strong cut-admissibility in canonical Gödel systems.

\section{Definition 11.}

1. A set $\mathcal{R}$ of canonical rules for an $n$-ary connective $\diamond$ is called coherent if $\mathcal{S} \cup \mathcal{S}_{1} \cup \mathcal{S}_{2}$ is classically inconsistent whenever $\mathcal{R}$ contains both $\mathcal{S} / \Rightarrow \diamond\left(p_{1}, \ldots, p_{n}\right)$ and $\mathcal{S}_{1}, \mathcal{S}_{2} / \diamond\left(p_{1}, \ldots, p_{n}\right) \Rightarrow$.

2. A canonical Gödel system $\mathbf{G}$ is called coherent if for each connective $\diamond$, the set of rules in $\mathbf{G}$ for $\diamond$ is coherent.

Note that every connective that was introduced in the examples above has a coherent set of rules.

\section{Many-Valued Semantics}

In this section we generalize many-valued semantics for Gödel logic to arbitrary coherent canonical Gödel systems. For this we introduce nondeterministic Gödel valuations.

Definition 12. A (non-deterministic) $\mathcal{L}$-Gödel valuation is a tuple $\mathcal{V}=\langle V, \leq, u\rangle$, where $\langle V, \leq\rangle$ is a nonempty linearly ordered set with a maximal element 1 and a minimal element 0 , and $u$ is a function from $\mathrm{Frm}_{\mathcal{L}}$ to $\mathrm{V}$.

Every canonical Gödel system $\mathbf{G}$ induces a set of $\mathcal{L}$-Gödel valuations for which it is sound and complete. We call the valuations of this set $\mathbf{G}$-legal valuations. The following definitions precisely define this set.

Notation. Given a nonempty linearly ordered set $\langle V, \leq\rangle$ with a maximal element 1 and a minimal element 0 , we denote by $\rightarrow$ the usual binary operation that corresponds to Gödel implication $(x \rightarrow y \doteq 1$ if $x \leq y$, otherwise $x \rightarrow y \doteq y)$.

Definition 13. Let $\mathcal{V}=\langle V, \leq, u\rangle$ be an $\mathcal{L}$-Gödel valuation. $^{1}$

- For every finite set of formulas $\Gamma$, $u_{\min }(\Gamma)=\min \{u(\psi) \mid \psi \in \Gamma\}, \quad$ and $u_{\max }(\Gamma)=\max \{u(\psi) \mid \psi \in \Gamma\}$.

- For every sequent $\Gamma \Rightarrow E, D_{\mathcal{V}}(\Gamma \Rightarrow E)=$ $u_{\min }(\Gamma) \rightarrow u_{\max }(E) . \quad D_{\mathcal{V}}$ is extended to sets of sequents by: $D_{\mathcal{V}}(\mathcal{S})=\min \left\{D_{\mathcal{V}}(s) \mid s \in \mathcal{S}\right\}$.

- For every set $\mathcal{S}$ of negative sequents, $N_{\mathcal{V}}(\mathcal{S})=$ $\max \left\{u_{\min }(\Gamma) \mid \Gamma \Rightarrow \in \mathcal{S}\right\}$.

Definition 14. Let $\mathcal{V}=\langle V, \leq, u\rangle$ be an $\mathcal{L}$-Gödel valuation.

- Let $r=\mathcal{S} / s$ be a right rule for an $n$-ary connective $\diamond$. $\mathcal{V}$ respects $r$ iff for every $\mathcal{L}$-substitution $\sigma, u\left(\sigma\left(\diamond\left(p_{1}, \ldots, p_{n}\right)\right) \geq D_{\mathcal{V}}(\sigma(\mathcal{S}))\right.$.

- Let $r=\mathcal{S}_{1}, \mathcal{S}_{2} / s$ be a left rule for an $n$ ary connective $\diamond$. $\mathcal{V}$ respects $r$ iff for every $\mathcal{L}$-substitution $\sigma, u\left(\sigma\left(\diamond\left(p_{1}, \ldots, p_{n}\right)\right) \leq\right.$ $D_{\mathcal{V}}\left(\sigma\left(\mathcal{S}_{1}\right)\right) \rightarrow N_{\mathcal{V}}\left(\sigma\left(\mathcal{S}_{2}\right)\right)$.

- Let $\mathbf{G}$ be a canonical Gödel system (for $\mathcal{L})$. $\mathcal{V}$ is $\mathbf{G}$-legal iff it respects the canonical rules of G.

Example 15 (Implication). Let $\mathcal{V}=\langle V, \leq, u\rangle$ be an $\mathcal{L}$-Gödel valuation. $\mathcal{V}$ respects the rule $(\Rightarrow \supset)$ iff $u(\varphi \supset \psi) \geq u(\varphi) \rightarrow u(\psi)$ for every $\varphi$ and $\psi$. $\mathcal{V}$ respects the rule $(\supset)$ iff $u(\varphi \supset \psi) \leq u(\varphi) \rightarrow u(\psi)$ for every $\varphi$ and $\psi$. The two conditions together imply the well-known Gödel semantics: $u(\varphi \supset \psi)=$ $u(\varphi) \rightarrow u(\psi)$.

Example 16 (Semi-Implication). Let $\mathcal{V}=\langle V, \leq, u\rangle$ be an $\mathcal{L}$-Gödel valuation. $\mathcal{V}$ respects the rule $\Leftrightarrow$ $\leadsto)$ iff $u(\varphi \leadsto \psi) \geq u(\psi)$ for every $\varphi$ and $\psi$. $\mathcal{V}$ respects the rule $(\leadsto)$ iff $u(\varphi \leadsto \psi) \leq u(\varphi) \rightarrow$

\footnotetext{
${ }^{1}$ We assume that $\min \emptyset=1$ and $\max \emptyset=0$.
} 
$u(\psi)$ for every $\varphi$ and $\psi$. Together we obtain the following non-deterministic semantics: $u(\varphi \sim \psi) \in$ $[u(\psi), u(\varphi) \rightarrow u(\psi)]$.

Example 17 (Asterisk). Let $\mathcal{V}=\langle V, \leq, u\rangle$ be an $\mathcal{L}$-Gödel valuation. $\mathcal{V}$ respects the rule $(\Rightarrow *)$ iff $u(\varphi * \psi) \geq \min \{u(\varphi), u(\psi)\}$ for every $\varphi$ and $\psi$. $\mathcal{V}$ respects the rule $(* \Rightarrow)$ iff $u(\varphi * \psi) \leq \max \{u(\varphi), u(\psi)\}$ for every $\varphi$ and $\psi$. Together we obtain the following non-deterministic semantics: $u(\varphi * \psi) \in$ $[\min \{u(\varphi), u(\psi)\}, \max \{u(\varphi), u(\psi)\}]$.

The semantic meaning of the coherence criterion is formulated in the next proposition.

Proposition 18. Let $\mathbf{G}$ be a canonical Gödel system (for $\mathcal{L})$, and $\mathcal{V}=\langle V, \leq, u\rangle$ be a $\mathbf{G}$-legal $\mathcal{L}$ Gödel valuation. Let $\mathcal{S} / \Rightarrow \diamond\left(p_{1}, \ldots, p_{n}\right)$ and $\mathcal{S}_{1}, \mathcal{S}_{2} / \diamond\left(p_{1}, \ldots, p_{n}\right) \Rightarrow$ be a right and a left rule for an $n$-ary connective $\diamond$. If $\mathbf{G}$ is coherent, then for every $\mathcal{L}$-substitution $\sigma, D_{\mathcal{V}}(\sigma(\mathcal{S})) \leq D_{\mathcal{V}}\left(\sigma\left(\mathcal{S}_{1}\right)\right) \rightarrow$ $N\left(\sigma\left(\mathcal{S}_{2}\right)\right)$.

Proof. Let $\sigma$ be an $\mathcal{L}$-substitution. Assume that $D_{\mathcal{V}}(\sigma(\mathcal{S}))>D_{\mathcal{V}}\left(\sigma\left(\mathcal{S}_{1}\right)\right) \rightarrow N_{\mathcal{V}}\left(\sigma\left(\mathcal{S}_{2}\right)\right)$. This implies that $D_{\mathcal{V}}(\sigma(\mathcal{S}))>N_{\mathcal{V}}\left(\sigma\left(\mathcal{S}_{2}\right)\right)$, and $D_{\mathcal{V}}\left(\sigma\left(\mathcal{S}_{1}\right)\right)>N_{\mathcal{V}}\left(\sigma\left(\mathcal{S}_{2}\right)\right)$. Consider the classical valuation on $p_{1}, \ldots, p_{n}$ such that $v\left(p_{i}\right)=t$ iff $u\left(\sigma\left(p_{i}\right)\right)>N_{\mathcal{V}}\left(\sigma\left(\mathcal{S}_{2}\right)\right)$. We prove that $v$ satisfies every clause in $\mathcal{S} \cup \mathcal{S}_{1} \cup \mathcal{S}_{2}$, and so $\mathbf{G}$ is not coherent.

Let $s=\Pi \Rightarrow E$ be a clause in $\mathcal{S} \cup \mathcal{S}_{1}$. Since $D_{\mathcal{V}}(\sigma(\mathcal{S}))>N_{\mathcal{V}}\left(\sigma\left(\mathcal{S}_{2}\right)\right)$ and $D_{\mathcal{V}}\left(\sigma\left(\mathcal{S}_{1}\right)\right)>$ $N_{\mathcal{V}}\left(\sigma\left(\mathcal{S}_{2}\right)\right), \quad D_{\mathcal{V}}(\sigma(s))>N_{\mathcal{V}}\left(\sigma\left(\mathcal{S}_{2}\right)\right)$, and so $u_{\text {min }}(\sigma(\Pi)) \rightarrow u_{\text {max }}(\sigma(E))>N_{\mathcal{V}}\left(\sigma\left(\mathcal{S}_{2}\right)\right)$. If $u_{\max }(\sigma(E))>N_{\mathcal{V}}\left(\sigma\left(\mathcal{S}_{2}\right)\right)$, it follows that $E=$ $\{p\}$ and $u(\sigma(p))>N_{\mathcal{V}}\left(\sigma\left(\mathcal{S}_{2}\right)\right)$, and so $v(p)=$ $t$. Thus $v$ classically satisfies $s$. Assume now that $u_{\max }(\sigma(E)) \leq N_{\mathcal{V}}\left(\sigma\left(\mathcal{S}_{2}\right)\right)$. This implies that $u_{\min }(\sigma(\Pi)) \leq u_{\max }(\sigma(E))$. It follows that $u_{\min }(\sigma(\Pi)) \leq N_{\mathcal{V}}\left(\sigma\left(\mathcal{S}_{2}\right)\right)$, and so there exists some $p \in \Pi$ such that $u(\sigma(p)) \leq N_{\mathcal{V}}\left(\sigma\left(\mathcal{S}_{2}\right)\right)$. Hence, $v(p)=f$ for some $p \in \Pi$. Thus $v$ classically satisfies $s$.

Now, let $s=\Pi \Rightarrow$ be a clause in $\mathcal{S}_{2}$. Obviously, $u_{\text {min }}(\sigma(\Pi)) \leq N_{\mathcal{V}}\left(\sigma\left(\mathcal{S}_{2}\right)\right)$. This implies that there exists some $p \in \Pi$ such that $u(\sigma(p)) \leq N_{\mathcal{V}}\left(\sigma\left(\mathcal{S}_{2}\right)\right)$, and so $v(p)=f$. Again $v$ classically satisfies $s$.

Next we define the many-valued semantic consequence relation between hypersequents which is induced by a canonical Gödel system.

\section{Definition 19.}

1. Let $\mathcal{V}=\langle V, \leq, u\rangle$ be an $\mathcal{L}$-Gödel valuation.

(a) $\mathcal{V}$ is a model of a sequent $\Gamma \Rightarrow E$ if $u_{\min }(\Gamma) \leq u_{\max }(E) \quad$ (equivalently, if $\left.D_{\mathcal{V}}(\Gamma \Rightarrow E)=1\right)$

(b) $\mathcal{V}$ is a model of a hypersequent $H$ if it is a model of one of its components. It is a model of a set $\mathcal{H}$ of hypersequents if it is a model of every $H \in \mathcal{H}$.
2. Let $\mathbf{G}$ be a canonical Gödel system, and let $\mathcal{H} \cup\{H\}$ be a set of hypersequents. $\mathcal{H} \vDash_{\mathbf{G}} H$ iff every $\mathbf{G}$-legal $\mathcal{L}$-Gödel valuation which is a model of $\mathcal{H}$ is also a model of $H$.

In general, in order for a denotational semantics of a propositional logic to be useful and effective, it should be analytic. This means that to determine whether a hypersequent $H$ follows from a set of hypersequents $\mathcal{H}$, it suffices to consider partial valuations, defined on the set of all subformulas of the formulas in $\mathcal{H} \cup\{H\}$. Now we show that the semantics of $\mathbf{G}$-legal $\mathcal{L}$-Gödel valuations is analytic in this sense.

Definition 20. Let $\mathcal{U}$ be a set of formulas closed under subformulas. A $\mathcal{U}$-Gödel semivaluation is a tuple $\mathcal{V}=\langle V, \leq, u\rangle$, where $\langle V, \leq\rangle$ are defined as in Definition 12 , and $u$ is a function from $\mathcal{U}$ to $V$.

Note that an $\mathcal{L}$-Gödel valuation is obtained by choosing $\mathcal{U}=\operatorname{Frm}_{\mathcal{L}}$. Definition 13 is applied for semivaluations without any changes. Definition 14 is modified as follows:

Definition 21. Let $\mathcal{V}=\langle V, \leq, u\rangle$ be a $\mathcal{U}$-Gödel semivaluation.

1 . Let $r=\mathcal{S} / s$ be a right rule for an $n$-ary connective $\diamond$. $\mathcal{V}$ respects $r$ iff for every $\mathcal{L}$-substitution $\sigma, u\left(\sigma\left(\diamond\left(p_{1}, \ldots, p_{n}\right)\right) \geq D_{\mathcal{V}}(\sigma(\mathcal{S}))\right.$ whenever $u\left(\sigma\left(\diamond\left(p_{1}, \ldots, p_{n}\right)\right)\right.$ is defined.

2. Let $r=\mathcal{S}_{1}, \mathcal{S}_{2} / s$ be a left rule for an $n$-ary connective $\diamond$. $\mathcal{V}$ respects $r$ iff for every $\mathcal{L}$-substitution $\sigma, u\left(\sigma\left(\diamond\left(p_{1}, \ldots, p_{n}\right)\right) \leq\right.$ $D_{\mathcal{V}}\left(\sigma\left(\mathcal{S}_{1}\right)\right) \quad \rightarrow \quad N_{\mathcal{V}}\left(\sigma\left(\mathcal{S}_{2}\right)\right), \quad$ whenever $u\left(\sigma\left(\diamond\left(p_{1}, \ldots, p_{n}\right)\right)\right.$ is defined.

3. Let $\mathbf{G}$ be a canonical Gödel system (for $\mathcal{L})$. $\mathcal{V}$ is $\mathbf{G}$-legal iff it respects the canonical rules of G.

Theorem 22 (Analyticity). Let $\mathbf{G}$ be a coherent canonical Gödel system for $\mathcal{L}$. The semantics of G-legal Gödel valuations is analytic in the following sense: For every set $\mathcal{U}$ of formulas closed under subformulas, if $\mathcal{V}=\langle V, \leq, u\rangle$ is a $\mathbf{G}$-legal $\mathcal{U}$-Gödel semivaluation, then $u$ can be extended to a function $u^{\prime}: \operatorname{Frm}_{\mathcal{L}} \rightarrow V$ so that $\mathcal{V}^{\prime}=\left\langle V, \leq, u^{\prime}\right\rangle$ is a $\mathbf{G}$-legal $\mathcal{L}$-Gödel valuation.

Proof. Let $\mathcal{U}$ be a set of formulas closed under subformulas, and let $\mathcal{V}=\langle V, \leq, u\rangle$ be a $\mathbf{G}$-legal $\mathcal{U}$ Gödel semivaluation. We recursively extend $u$ to a total function $u^{\prime}$. For atomic $p$ we let $u^{\prime}(p)=$ $u(p)$ if $u(p)$ is defined, and $u^{\prime}(p)=1$ (say) otherwise. For $\varphi=\diamond\left(\psi_{1}, \ldots, \psi_{n}\right)$ we let $u^{\prime}(\varphi)=$ $u(\varphi)$ whenever $u(\varphi)$ is defined, and otherwise we define $u^{\prime}(\varphi)=\max \left\{D_{\mathcal{V}^{\prime}}\left(\sigma_{\psi_{1}, \ldots, \psi_{n}}(\mathcal{S})\right) \mid \mathcal{S} / \Rightarrow\right.$ $\left.\diamond\left(p_{1}, \ldots, p_{n}\right) \in R_{\diamond}\right\}$, where $R_{\diamond}$ denotes the set of right rules for $\diamond$ in $\mathbf{G}$. Note that the value of $D_{\mathcal{V}^{\prime}}\left(\sigma_{\psi_{1}, \ldots, \psi_{n}}(\mathcal{S})\right)$ depends only on the values assigned by $u^{\prime}$ to $\psi_{1}, \ldots, \psi_{n}$, so the recursion works, and $u^{\prime}$ is well defined. 
From the definition of $u^{\prime}$, it immediately follows that $u^{\prime}$ is an extension of $u$. It remains to show that $\mathcal{V}^{\prime}=\left\langle V, \leq, u^{\prime}\right\rangle$ is $\mathbf{G}$-legal, i.e. that $\mathcal{V}^{\prime}$ respects the rules of $\mathbf{G}$.

Let $r=\mathcal{S} / \Rightarrow \diamond\left(p_{1}, \ldots, p_{n}\right)$ be a right rule for an $n$-ary connective $\diamond$, and let $\sigma$ be an $\mathcal{L}$-substitution. If $u\left(\sigma\left(\diamond\left(p_{1}, \ldots, p_{n}\right)\right)\right)$ is defined, then since $\mathcal{U}$ is closed under subformulas, for every $1 \leq i \leq n, u\left(\sigma\left(p_{i}\right)\right)$ is defined. In this case, our construction ensures that for every $1 \leq i \leq n$ we have $u^{\prime}\left(\sigma\left(p_{i}\right)\right)=u\left(\sigma\left(p_{i}\right)\right)$, and it easily follows that $D_{\mathcal{V}}(\sigma(\mathcal{S}))=D_{\mathcal{V}^{\prime}}(\sigma(\mathcal{S}))$. Since $\mathcal{V}$ respects $r, u\left(\diamond\left(\psi_{1}, \ldots, \psi_{n}\right)\right) \geq D_{\mathcal{V}}(\sigma(\mathcal{S}))$, and so $u^{\prime}\left(\diamond\left(\psi_{1}, \ldots, \psi_{n}\right)\right) \geq D_{\mathcal{V}^{\prime}}(\sigma(\mathcal{S}))$. Assume now that $u\left(\sigma\left(\diamond\left(p_{1}, \ldots, p_{n}\right)\right)\right)$ is not defined, and so $u^{\prime}\left(\sigma\left(\diamond\left(p_{1}, \ldots, p_{n}\right)\right)\right)=\max B$, where $B=$ $\left\{D_{\mathcal{V}^{\prime}}(\sigma(\mathcal{S})) \mid \mathcal{S} / \Rightarrow \diamond\left(p_{1}, \ldots, p_{n}\right) \in R_{\diamond}\right\}$. Obviously, $u^{\prime}\left(\sigma\left(\diamond\left(p_{1}, \ldots, p_{n}\right)\right)\right)=\max B \geq D_{\mathcal{V}^{\prime}}(\sigma(\mathcal{S}))$.

Now, let $r=\mathcal{S}_{1}, \mathcal{S}_{2} / \Rightarrow \diamond\left(p_{1}, \ldots, p_{n}\right)$ be a left rule for an $n$-ary connective $\diamond$, and let $\sigma$ be an $\mathcal{L}$-substitution. As for the right rules, if $u\left(\sigma\left(\diamond\left(p_{1}, \ldots, p_{n}\right)\right)\right)$ is defined, then since $\mathcal{U}$ is closed under subformulas and $\mathcal{V}$ is $\mathbf{G}$-legal, it follows that $u^{\prime}\left(\sigma\left(\diamond\left(p_{1}, \ldots, p_{n}\right)\right) \leq D_{\mathcal{V}^{\prime}}\left(\sigma\left(\mathcal{S}_{1}\right)\right) \rightarrow N_{\mathcal{V}^{\prime}}\left(\sigma\left(\mathcal{S}_{2}\right)\right)\right.$. Assume now that $u\left(\sigma\left(\diamond\left(p_{1}, \ldots, p_{n}\right)\right)\right)$ is not defined, and so $u^{\prime}\left(\sigma\left(\diamond\left(p_{1}, \ldots, p_{n}\right)\right)\right)=\max B$, where $B=\left\{D_{\mathcal{V}^{\prime}}(\sigma(\mathcal{S})) \mid \mathcal{S} / \Rightarrow \diamond\left(p_{1}, \ldots, p_{n}\right) \in R_{\diamond}\right\}$. If $R_{\diamond}$ is empty then $u^{\prime}\left(\sigma\left(\diamond\left(p_{1}, \ldots, p_{n}\right)\right)\right)=0$ and obviously $0 \leq D_{\mathcal{V}^{\prime}}\left(\sigma\left(\mathcal{S}_{1}\right)\right) \rightarrow N_{\mathcal{V}^{\prime}}\left(\sigma\left(\mathcal{S}_{2}\right)\right)$. Otherwise, there exists a rule $\mathcal{S} / \Rightarrow \diamond\left(p_{1}, \ldots, p_{n}\right) \in$ $R_{\diamond}$ such that $u^{\prime}\left(\sigma\left(\diamond\left(p_{1}, \ldots, p_{n}\right)\right)\right)=D_{\mathcal{V}^{\prime}}(\sigma(\mathcal{S}))$. Since $\mathbf{G}$ is coherent, Proposition 18 implies that $D_{\mathcal{V}}(\sigma(\mathcal{S})) \leq D_{\mathcal{V}}\left(\sigma\left(\mathcal{S}_{1}\right)\right) \rightarrow N\left(\sigma\left(\mathcal{S}_{2}\right)\right)$. It follows that $u^{\prime}\left(\sigma\left(\diamond\left(p_{1}, \ldots, p_{n}\right)\right)\right) \leq D_{\mathcal{V}}\left(\sigma\left(\mathcal{S}_{1}\right)\right) \rightarrow$ $N\left(\sigma\left(\mathcal{S}_{2}\right)\right)$, and so $\mathcal{V}^{\prime}$ respects $r$.

It remains to prove soundness and completeness of the semantics of $\mathbf{G}$-legal Gödel valuations. We shall return to this in Section 6 .

\section{Kripke-style Semantics}

It is well-known that Gödel logic is also characterized as the logic of linear intuitionistic Kripke frames. In this section we generalize this semantics to arbitrary coherent canonical Gödel systems. We prove that every coherent canonical Gödel system induces a set of non-deterministic linear Kripkeframes for which it is sound and complete. The semantics is then used to show that every coherent canonical Gödel system enjoys strong cutadmissibility.

The following definitions are "the linear version" of the general intuitionistic-like Kripke semantics presented in [4] for arbitrary coherent canonical sequent systems. The reader is referred to [4] for more details.

Definition 23. Let $\langle W, \leq\rangle$ be a nonempty partially ordered set. Let $\mathcal{U}$ be a set of formulas. A function $v: W \times \mathcal{U} \rightarrow\{t, f\}$ is called persistent iff for every $a \in W$ and $\varphi \in \mathcal{U}, v(a, \varphi)=t$ implies that $v(b, \varphi)=$ $t$ for every $b \in W$ such that $a \leq b$.

Definition 24. Let $\mathcal{U}$ be a set of formulas closed under subformulas. A $\mathcal{U}$-Gödel semiframe is a triple $\mathcal{W}=\langle W, \leq, v\rangle$ such that:

1. $\langle W, \leq\rangle$ is a nonempty linearly ordered set.

2. $v$ is a persistent function from $W \times \mathcal{U}$ to $\{t, f\}$.

When $\mathcal{U}=\operatorname{Frm}_{\mathcal{L}}$ a $\mathcal{U}$-Gödel semiframe is also called an $\mathcal{L}$-Gödel frame.

Definition 25. Let $\mathcal{W}=\langle W, \leq, v\rangle$ be a $\mathcal{U}$-Gödel semiframe.

1. A sequent $\Gamma \Rightarrow E$ is locally true in $a \in W$ iff $\Gamma \cup E \subseteq \mathcal{U}$, and either $v(a, \psi)=f$ for some $\psi \in \Gamma$, or $E=\{\varphi\}$ and $v(a, \varphi)=t$.

2. A sequent is true in $a \in W$ iff it is locally true in every $b \geq a$.

Definition 26. Let $\mathcal{W}=\langle W, \leq, v\rangle$ be a $\mathcal{U}$-Gödel semiframe.

1. An $\mathcal{L}$-substitution $\sigma$ (locally) satisfies a sequent $\Gamma \Rightarrow E$ in $a \in W$ if $\sigma(\Gamma) \Rightarrow \sigma(E)$ is (locally) true in $a$.

2. An $\mathcal{L}$-substitution fulfils a right rule $\mathcal{S} / s$ in $a \in$ $W$ if it satisfies in a every clause in $\mathcal{S}$.

3. An $\mathcal{L}$-substitution fulfils a left rule $\mathcal{S}_{1}, \mathcal{S}_{2} / s$ in $a \in W$ if it satisfies in $a$ every clause in $\mathcal{S}_{1}$, and locally satisfies in a every clause in $\mathcal{S}_{2}$.

4. Let $r$ be a canonical rule for an $n$-ary connective $\diamond$. $\mathcal{W}$ respects $r=\mathcal{S} / s$ or $r=\mathcal{S}_{1}, \mathcal{S}_{2} / s$ if for every $a \in W$ and every substitution $\sigma$ : if $\sigma$ fulfils $r$ in $a$ and $\sigma\left(\diamond\left(p_{1}, \ldots, p_{n}\right)\right) \in \mathcal{U}$ then $\sigma$ locally satisfies $s$ in $a$.

5. Let $\mathbf{G}$ be a canonical Gödel system. $\mathcal{W}$ is $\mathbf{G}$ legal iff it respects the canonical rules of $\mathbf{G}$.

Example 27 (Implication). An $\mathcal{L}$-Gödel frame $\mathcal{W}=\langle W, \leq, v\rangle$ respects the rule $(\supset \Rightarrow)$ iff for every $a \in W, v(a, \varphi \supset \psi)=f$ whenever $v(b, \varphi)=t$ for every $b \geq a$ and $v(a, \psi)=f$. Because of the persistence condition, this is equivalent to $v(a, \varphi \supset \psi)=$ $f$ whenever $v(a, \varphi)=t$ and $v(a, \psi)=f$. Again by the persistence condition, $v(a, \varphi \supset \psi)=f$ iff $v(b, \varphi \supset \psi)=f$ for some $b \geq a$. Hence, we get: $v(a, \varphi \supset \psi)=f$ whenever there exists $b \geq a$ such that $v(b, \varphi)=t$ and $v(b, \psi)=f$. $\mathcal{W}$ respects $(\Rightarrow \supset)$ iff for every $a \in W, v(a, \varphi \supset \psi)=t$ whenever for every $b \geq a$, either $v(b, \varphi)=f$ or $v(b, \psi)=t$. Hence the two rules together impose exactly the well-known Kripke semantics for implication.

Example 28 (Semi-Implication). An $\mathcal{L}$-Gödel frame $\mathcal{W}=\langle W, \leq, v\rangle$ respects the rule $(\leadsto)$ under the same conditions it respects $(\supset)$. $\mathcal{W}$ respects $(\Longrightarrow)$ iff for every $a \in W, v(a, \varphi \leadsto \psi)=t$ whenever $v(a, \psi)=t$ (recall that this is equivalent to $v(b, \psi)=t$ for every $b \geq a)$. Note that in this case the two rules for $\leadsto$ do not always determine the value assigned to $\varphi \sim \psi$ : if $v(a, \psi)=f$, and there 
is no $b \geq a$ such that $v(b, \varphi)=t$ and $v(b, \psi)=f$, then $v(a, \varphi \leadsto \psi)$ is free to be either $t$ or $f$. So the Kripke-style semantics of this connective is nondeterministic.

Example 29 (Asterisk). An $\mathcal{L}$-Gödel frame $\mathcal{W}=$ $\langle W, \leq, v\rangle$ respects the rule $(* \Rightarrow)$ iff $v(a, \varphi * \psi)=f$ whenever $v(a, \varphi)=f$ and $v(a, \psi)=f$. It respects $(\Rightarrow *)$ iff $v(a, \varphi * \psi)=t$ whenever $v(a, \varphi)=t$ and $v(a, \psi)=t$. Again, the Kripke-style semantics of this connective is non-deterministic.

Next we define the Kripke-style semantic consequence relation between hypersequents which is induced by a canonical Gödel system.

\section{Definition 30.}

1. Let $\mathcal{W}=\langle W, \leq, v\rangle$ be a $\mathcal{U}$-Gödel semiframe.

(a) $\mathcal{W}$ is a model of a sequent $s$ iff $s$ is locally true in every $a \in W$.

(b) $\mathcal{W}$ is a model of a hypersequent $H$ if it is a model of at least one of its components. It is a model of a set of hypersequents $\mathcal{H}$ if it is a model of every $H \in \mathcal{H}$.

2. Let $\mathbf{G}$ be a canonical Gödel system, and let $\mathcal{H} \cup\{H\}$ be a set of hypersequents. $\mathcal{H} \vDash_{\mathbf{G}}^{K} H$ iff every $\mathbf{G}$-legal $\mathcal{L}$-Gödel frame which is a model of $\mathcal{H}$ is also a model of $H$.

Again, we show that this semantics is analytic, and so to decide whether $\mathcal{H} \vDash_{\mathbf{G}}^{K} H$, it suffices to consider semiframes, defined on the set of all subformulas of the formulas in $\mathcal{H} \cup\{H\}$.

Theorem 31 (Analyticity). Let $\mathbf{G}$ be a coherent canonical Gödel system for $\mathcal{L}$. The semantics of $\mathbf{G}$-legal Gödel frames is analytic in the following sense: For every set $\mathcal{U}$ of formulas closed under subformulas, if $\mathcal{W}=\langle W, \leq, v\rangle$ is a $\mathbf{G}$-legal $\mathcal{U}$-Gödel semiframe, then $v$ can be extended to a function $v^{\prime}: W \times \operatorname{Frm}_{\mathcal{L}} \rightarrow\{t, f\}$ so that $\mathcal{W}^{\prime}=\left\langle W, \leq, v^{\prime}\right\rangle$ is a $\mathbf{G}$-legal $\mathcal{L}$-Gödel frame.

Proof. Directly follows from Theorem 7.1 in [4] (the linearity of $\leq$ is not used).

Next we turn to soundness and completeness of this Kripke-style semantics.

Theorem 32. Let $\mathbf{G}$ be a coherent canonical Gödel system, and let $\mathcal{H} \cup\{H\}$ be a set of hypersequents. If $\mathcal{H} \vdash_{\mathbf{G}} H$ then $\mathcal{H} \vDash_{\mathbf{G}}^{K} H$.

Proof. Straight-forward modification of the proof of Theorem 6.1 in [4]. Note that the soundness of the communication rule follows from the linearity of the order relation.

Theorem 33. Let $\mathbf{G}$ be a coherent canonical Gödel system, and let $\mathcal{H} \cup\{H\}$ be a set of hypersequents. Then either $\mathcal{H} \vdash_{\mathbf{G}}^{\text {frm }[\mathcal{H}]} H$, or there is a $\mathbf{G}$-legal $\mathcal{L}$ Gödel frame which is a model of $\mathcal{H}$ but not a model of $H$.
Proof. (Outline) $)^{2}$

Assume $\mathcal{H} \nvdash_{\mathbf{G}}^{\text {frm }[\mathcal{H}]} H$. We construct a $\mathbf{G}$-legal $\mathcal{L}$ Gödel frame $\mathcal{W}=\langle W, \leq, v\rangle$, which is a model of $\mathcal{H}$ but not a model of $H$.

Let $\mathcal{F}=\operatorname{sub}[\mathcal{H} \cup\{H\}]$. It is a standard matter to construct a (possibly infinite) set of sequents $\Omega$ such that: (i) $H \subseteq \Omega$; (ii) frm $[\Omega] \subseteq \mathcal{F}$; (iii) For every hypersequent $H^{\prime} \subseteq \Omega, \mathcal{H} \forall_{\mathbf{G}}^{f r m[\mathcal{H}]} H^{\prime}$; and $(i v)$ For every sequent $s \notin \Omega$, there exists a hypersequent $H^{\prime} \subseteq \Omega$, such that $\mathcal{H} \vdash_{\mathbf{G}}^{\text {frm }[\mathcal{H}]} H^{\prime} \mid s$.

Let $\mathcal{F}^{\prime}=\{E \subseteq \mathcal{F} \mid \exists \Gamma \subseteq \mathcal{F} .(\Gamma \Rightarrow E) \in \Omega\}$. For $E \in \mathcal{F}^{\prime}$ let $\mathcal{T}_{E}$ be the union of all the sets $\Gamma$ such that $(\Gamma \Rightarrow E) \in \Omega$. In the presence of the communication rule it can be now proved that $(*)$ for every $E \in \mathcal{F}^{\prime}$ and $\Gamma \subseteq \mathcal{F},(\Gamma \Rightarrow E) \in \Omega$ iff $\Gamma \subseteq \mathcal{T}_{E}$.

Define $W=\left\{\mathcal{T}_{E} \mid E \in \mathcal{F}^{\prime}\right\}$ and $\leq=\subseteq$. Using (*) and the communication rule, it can be proved that $\leq$ is a linear order on $W$. The valuation $v: W \times \operatorname{Frm}_{\mathcal{L}} \rightarrow\{t, f\}$ is inductively defined as follows: for atomic formulas, $v(\mathcal{T}, p)=t$ iff $p \in \mathcal{T}$. Suppose $v\left(\mathcal{T}, \psi_{i}\right)$ has been defined for every $\mathcal{T} \in W$ and $1 \leq i \leq n$. We let $v\left(\mathcal{T}, \diamond\left(\psi_{1}, \ldots, \psi_{n}\right)\right)=t$ iff at least one of the following holds:

1. There exists a right rule for $\diamond$ which is fulfilled in $\mathcal{T}$ by $\sigma_{\psi_{1}, \ldots, \psi_{n}}$.

2. $\diamond\left(\psi_{1}, \ldots, \psi_{n}\right) \in \mathcal{T}$ and there does not exist $\mathcal{T}^{\prime} \in W, \mathcal{T} \subseteq \mathcal{T}^{\prime}$, and a left rule for $\diamond$ which is fulfilled in $\mathcal{T}^{\prime}$ by $\sigma_{\psi_{1}, \ldots, \psi_{n}}$.

It can be now proved that $v$ is persistent, and so $\mathcal{W}$ is an $\mathcal{L}$-Gödel frame. Moreover, using $\mathbf{G}$ 's coherence property, one proves that $\mathcal{W}$ is $\mathbf{G}$-legal. It remains to show that $\mathcal{W}$ is a model of $\mathcal{H}$ but not a model of $H$. This follows from the following two properties: for every $\mathcal{T} \in W$ and every formula $\psi \in \mathcal{F}$ : (a) If $\psi \in \mathcal{T}$ then $v(\mathcal{T}, \psi)=t$; (b) If $\mathcal{T}=\mathcal{T}_{\psi}$ then $v(\mathcal{T}, \psi)=f$. (a) and (b) are proved together by a simultaneous induction on the complexity of $\psi$.

The following are corollaries of theorems 32 and $33:$

Corollary 34 (Soundness and Completeness). Every coherent canonical Gödel system $\mathbf{G}$ is strongly sound and complete with respect to the semantics of $\mathbf{G}$-legal Gödel frames. In other words: $\mathcal{H} \vdash_{\mathbf{G}} H$ iff $\mathcal{H} \vDash \underset{\mathbf{G}}{K} H$.

Corollary 35. The following conditions are equivalent for a canonical Gödel system $\mathbf{G}$ :

1. $\left(\Rightarrow p_{1}\right),\left(p_{2} \Rightarrow\right) \forall \mathbf{G} \Rightarrow$.

2. $\mathbf{G}$ is coherent.

3. $\mathbf{G}$ enjoys strong cut-admissibility.

Proof. 1 implies 2 by Theorem 4.10 in [4] (note that its proof does not involve hypersequents). 2 implies

\footnotetext{
${ }^{2}$ The proof is a generalization of the semantic proof given in [2] of cut-admissibility in the usual hypersequential calculus for propositional Gödel logic.
} 
3 by Theorem 33 and Corollary 34. Finally, without using cuts on formulas different from $p_{1}$ and $p_{2}$, there is no way to derive $\Rightarrow$ from $\Rightarrow p_{1}$ and $p_{2} \Rightarrow$ in a canonical Gödel system. Hence 3 implies 1 .

Corollary 36. Let $\mathbf{G}$ be a coherent canonical Gödel system. Then $\mathbf{G}$ is strongly decidable: Given a finite set $\mathcal{H}$ of hypersequents, and a hypersequent $H$, it is decidable whether $\mathcal{H} \vdash_{\mathbf{G}} H$ or not.

\section{Soundness and Completeness with respect to the Many-valued Semantics}

In this section we show that the Kripke-style semantics introduced in the previous section is equivalent to the many-valued semantics introduced in Section 4. As a corollary, we establish the soundness and completeness of coherent canonical Gödel systems with respect to their induced many-valued semantics.

Lemma 37. Let $\mathcal{V}=\langle V, \leq, u\rangle$ be an $\mathcal{L}$-Gödel valuation. Let $\mathcal{W}=\langle V, \geq, v\rangle$, where $v: V \times$ Frm $_{\mathcal{L}} \rightarrow$ $\{t, f\}$ is defined as follows:

$$
v(a, \psi)= \begin{cases}t & u(\psi) \neq 0 \text { and } u(\psi) \geq a \\ f & \text { otherwise }\end{cases}
$$

1. $\mathcal{W}$ is an $\mathcal{L}$-Gödel frame.

2. If $\Gamma \Rightarrow$ is locally true in $a \in V$ and $a \neq 0$ then $u_{\min }(\Gamma)<a$.

3. If $\Gamma \Rightarrow$ is locally true in 0 then $u_{\min }(\Gamma)=0$.

4. If a sequent $s$ is true in $a \in V$ and $a \neq 0$ then $a \leq D_{\mathcal{V}}(s)$.

5. If a sequent $s$ is true in 0 then $D_{\mathcal{V}}(s)>0$.

6. Let $\mathbf{G}$ be a canonical Gödel system. If $\mathcal{V}$ is $\mathbf{G}$-legal then $\mathcal{W}$ is $\mathbf{G}$-legal.

7. $\mathcal{W}$ is a model of a hypersequent $H$ iff $\mathcal{V}$ is a model of $H$.

Proof. 1. Obviously $\langle V, \geq\rangle$ is a non-empty linearly ordered set. It is also easy to see that $v$ is persistent.

2. Assume $\Gamma \Rightarrow$ is locally true in $a \neq 0$. Thus there exists $\psi \in \Gamma$ such that $v(a, \psi)=f$. This implies that $u(\psi)=0$ or $u(\psi)<a$. Since $a \neq 0$, $u(\psi)<a$ in any case. $u_{\min }(\Gamma) \leq u(\psi)$, and so $u_{\min }(\Gamma)<a$.

3. Assume $\Gamma \Rightarrow$ is locally true in 0 . Thus there exists $\psi \in \Gamma$ such that $v(0, \psi)=f$. This implies that $u(\psi)=0$, and so $u_{\min }(\Gamma)=0$.

4. Let $s=\Gamma \Rightarrow E$. Assume that $s$ is true in $a$. Thus for every $b \leq a$, there either exists $\psi \in \Gamma$ such that $v(b, \psi)=f$ or $E=\{\varphi\}$ and $v(b, \varphi)=$ $t$. This implies that for every $b \leq a$, either (1) there exists $\psi \in \Gamma$ such that $u(\psi)=0$ or $u(\psi)<b$, or $(2) E=\{\varphi\}, u(\varphi) \neq 0$ and $u(\varphi) \geq$ $b$. Hence, for every $b \leq a$, either $(1) u_{\min }(\Gamma)=$ 0 or $u_{\min }(\Gamma)<b$, or $(2) u_{\max }(E) \neq 0$ and $u_{\text {max }}(E) \geq b$. It is easy to prove that for every $x, y \in V$, if $\forall b \leq a .(x=0) \vee(x<b) \vee((y \neq$ $0) \wedge(y \geq b))$ then $a \leq x \rightarrow y$. It follows that $a \leq u_{\min }(\Gamma) \rightarrow u_{\max }(E)$, and so $a \leq D_{\mathcal{V}}(s)$.
5. Let $s=\Gamma \Rightarrow E$. Assume that $s$ is true in 0 . Thus there either exists $\psi \in \Gamma$ such that $v(0, \psi)=f$ or $E=\{\varphi\}$ and $v(0, \varphi)=t$. This implies that either there exists $\psi \in \Gamma$ such that $u(\psi)=0$ or $E=\{\varphi\}$ and $u(\varphi) \neq 0$. Hence, either $u_{\min }(\Gamma)=0$, or $u_{\max }(E) \neq 0$. It follows that $u_{\min }(\Gamma) \rightarrow u_{\max }(E)>0$, and so $D_{\mathcal{V}}(s)>0$.

6. We show that $\mathcal{W}$ respects the rules of $\mathbf{G}$. Let $r=\mathcal{S} / \Rightarrow \diamond\left(p_{1}, \ldots, p_{n}\right) \quad\left(r=\mathcal{S}_{1}, \mathcal{S}_{2} / \diamond\right.$ $\left.\left(p_{1}, \ldots, p_{n}\right) \Rightarrow\right)$ be a rule in $\mathbf{G}$ for an $n$-ary connective $\diamond$. Let $a \in V$, and let $\sigma$ be an $\mathcal{L}$-substitution. Suppose that $\sigma$ fulfils $r$ in $a$, we prove that $\sigma$ satisfies $\Rightarrow \diamond\left(p_{1}, \ldots, p_{n}\right)$ $\left(\diamond\left(p_{1}, \ldots, p_{n}\right) \Rightarrow\right)$ in $a$.

First, suppose that $r$ is a right rule. We show that $u\left(\sigma\left(\diamond\left(p_{1}, \ldots, p_{n}\right)\right)\right) \neq 0$ and $u\left(\sigma\left(\diamond\left(p_{1}, \ldots, p_{n}\right)\right)\right) \geq a$ (and so $\left.v\left(a, \sigma\left(\diamond\left(p_{1}, \ldots, p_{n}\right)\right)\right)=t\right) . \quad$ Consider two cases:

(i) $\mathbf{a}>\mathbf{0}$ : Since $\sigma(s)$ is true in $a$ for every $s \in \mathcal{S}, a \leq D_{\mathcal{V}}(\sigma(s))$ for every $s \in \mathcal{S}$. It follows that $a \leq D_{\mathcal{V}}(\sigma(\mathcal{S}))$. Since $\mathcal{V}$ is $\mathbf{G}$-legal, $u\left(\sigma\left(\diamond\left(p_{1}, \ldots, p_{n}\right)\right) \geq D_{\mathcal{V}}(\sigma(\mathcal{S})) \geq a>0\right.$.

(ii) $\mathbf{a}=\mathbf{0}$ : Since $\sigma(s)$ is true in $a$ for every $s \in \mathcal{S}, D_{\mathcal{V}}(\sigma(s))>0$ for every $s \in \mathcal{S}$. It follows that $D_{\mathcal{V}}(\sigma(\mathcal{S}))>0$. Since $\mathcal{V}$ is G-legal, $u\left(\sigma\left(\diamond\left(p_{1}, \ldots, p_{n}\right)\right) \geq D_{\mathcal{V}}(\sigma(\mathcal{S}))>0=a\right.$.

Second, suppose that $r$ is a left rule. We show that $u\left(\sigma\left(\diamond\left(p_{1}, \ldots, p_{n}\right)\right)\right)=0$ or $u\left(\sigma\left(\diamond\left(p_{1}, \ldots, p_{n}\right)\right)\right)<a$ (and so $\left.v\left(a, \sigma\left(\diamond\left(p_{1}, \ldots, p_{n}\right)\right)\right)=f\right)$. Again, consider two cases:

(i) $\mathbf{a}>\mathbf{0}$ : Since $\sigma(s)$ is true in $a$ for every $s \in$ $\mathcal{S}_{1}$, it follows that $a \leq D_{\mathcal{V}}\left(\sigma\left(\mathcal{S}_{1}\right)\right)$. Since $\sigma(\Pi \Rightarrow$ ) is locally true in $a$ for every $(\Pi \Rightarrow) \in \mathcal{S}_{2}$, $u_{\text {min }}(\sigma(\Pi))<a$ for every $(\Pi \Rightarrow) \in \mathcal{S}_{2}$. It follows that $N_{\mathcal{V}}\left(\sigma\left(\mathcal{S}_{2}\right)\right)<a$. Since $\mathcal{V}$ is $\mathbf{G}$-legal, $u\left(\sigma\left(\diamond\left(p_{1}, \ldots, p_{n}\right)\right) \leq D_{\mathcal{V}}\left(\sigma\left(\mathcal{S}_{1}\right)\right) \rightarrow N_{\mathcal{V}}\left(\sigma\left(\mathcal{S}_{2}\right)\right)\right.$. Together it follows that $u\left(\sigma\left(\diamond\left(p_{1}, \ldots, p_{n}\right)\right)<a\right.$. (ii) $\mathbf{a}=\mathbf{0}$ : Since $\sigma(s)$ is true in 0 for every $s \in \mathcal{S}_{1}$, it follows that $D_{\mathcal{V}}\left(\sigma\left(\mathcal{S}_{1}\right)\right)>0$. Since $\sigma(\Pi \Rightarrow)$ is locally true in 0 for every $(\Pi \Rightarrow) \in \mathcal{S}_{2}, u_{\min }(\sigma(\Pi))=0$ for every $(\Pi \Rightarrow) \in \mathcal{S}_{2}$. It follows that $N_{\mathcal{V}}\left(\sigma\left(\mathcal{S}_{2}\right)\right)=$ 0. Since $\mathcal{V}$ is G-legal, $u\left(\sigma\left(\diamond\left(p_{1}, \ldots, p_{n}\right)\right) \leq\right.$ $D_{\mathcal{V}}\left(\sigma\left(\mathcal{S}_{1}\right)\right) \rightarrow N_{\mathcal{V}}\left(\sigma\left(\mathcal{S}_{2}\right)\right)=0$. It follows that $u\left(\sigma\left(\diamond\left(p_{1}, \ldots, p_{n}\right)\right)=0\right.$.

7. We prove one direction. The converse is left to the reader. Assume that $\mathcal{W}$ is a model of some hypersequent $H$. Thus there exists a sequent $s \in H$ such that $\mathcal{W}$ is a model of $s$. In particular, $s$ is true in 1 , and by $4, D_{\mathcal{V}}(s)=1$. It follows that $\mathcal{V}$ is a model of $s$, and so $\mathcal{V}$ is a model of $H$.

Lemma 38. Let $\mathcal{W}=\langle W, \leq, v\rangle$ be an $\mathcal{L}$-Gödel frame. Let $\mathcal{V}=\langle V, \subseteq, u\rangle$, where $V$ is the set of all 
upwards closed subsets of $W{ }^{3}$ and $u:$ Frm $_{\mathcal{L}} \rightarrow V$ such that $u(\psi)=\{w \in W \mid v(w, \psi)=t\}$.

1. $\mathcal{V}$ is an $\mathcal{L}$-Gödel valuation.

2. If $a \in D_{\mathcal{V}}(\Gamma \Rightarrow E)$ then $\Gamma \Rightarrow E$ is true in $a$.

3. If $a \notin u_{\min }(\Gamma)$ then $\Gamma \Rightarrow$ is locally true in $a$.

4. Let $\mathbf{G}$ be a canonical Gödel system. If $\mathcal{W}$ is $\mathbf{G}$-legal then $\mathcal{V}$ is $\mathbf{G}$-legal.

5. $\mathcal{V}$ is a model of a hypersequent $H$ iff $\mathcal{W}$ is a model of $H$.

Proof. 1. It is easy to see that $\langle V, \subseteq\rangle$ is a nonempty linearly ordered set with a maximal element $1=W$ and a minimal element $0=\emptyset$.

2. Let $s=\Gamma \Rightarrow E$. If $a \in D_{\mathcal{V}}(s)$, either $u_{\text {min }}(\Gamma) \subseteq$ $u_{\max }(E)$ or $a \in u_{\max }(E)$. Consider two cases:

$E=\emptyset$ In this case $u_{\max }(E)=\emptyset$, and so $u_{\min }(\Gamma)=\emptyset$. It follows that there exists some $\psi \in \Gamma$ such that $v(b, \psi)=f$ for every $b \in W$. In particular $s$ is true in $a$.

$E \neq \emptyset$ Assume $E=\{\varphi\}$, and so $u_{\max }(E)=$ $u(\varphi)$. If $a \in u(\varphi)$ then $v(a, \varphi)=t$ and $s$ is true in $a$. Otherwise, $u_{\min }(\Gamma) \subseteq u(\varphi)$. It implies that $u(\psi) \subseteq u(\varphi)$ for some $\psi \in \Gamma$. Therefore, $s$ is locally true in every $b \in W$, and so $s$ is true in $a$.

3. Since $a \notin u_{\min }(\Gamma)$, there exists $\psi \in \Gamma$ such that $a \notin u(\psi)$. It follows from the definition of $u$, that $v(a, \psi)=f$, and so $s$ is locally true in $a$.

4. We show that $\mathcal{V}$ respects the rules of $\mathbf{G}$. Let $r=\mathcal{S} / \Rightarrow \diamond\left(p_{1}, \ldots, p_{n}\right)\left(\mathcal{S}_{1}, \mathcal{S}_{2} / \diamond\left(p_{1}, \ldots, p_{n}\right) \Rightarrow\right)$ be a rule in $\mathbf{G}$ for an $n$-ary connective $\diamond$. Let $\sigma$ be an $\mathcal{L}$-substitution.

First, suppose that $r$ is a right rule. We prove that $D_{\mathcal{V}}(\sigma(\mathcal{S})) \subseteq u\left(\sigma\left(\diamond\left(p_{1}, \ldots, p_{n}\right)\right)\right.$. Let $a \in$ $D_{\mathcal{V}}(\sigma(\mathcal{S}))$. By 2, every $s \in \sigma(\mathcal{S})$ is true in $a$. It follows that $\sigma$ fulfils $r$ in $a$. Since $\mathcal{W}$ is G-legal, $v\left(a, \sigma\left(\diamond\left(p_{1}, \ldots, p_{n}\right)\right)\right)=t$, and so $a \in u\left(\sigma\left(\diamond\left(p_{1}, \ldots, p_{n}\right)\right)\right)$.

Second, suppose that $r$ is a left rule. We show that $u\left(\sigma\left(\diamond\left(p_{1}, \ldots, p_{n}\right)\right) \subseteq\right.$ $D_{\mathcal{V}}\left(\sigma\left(\mathcal{S}_{1}\right)\right) \rightarrow N_{\mathcal{V}}\left(\sigma\left(\mathcal{S}_{2}\right)\right)$. This obviously holds if $D_{\mathcal{V}}\left(\sigma\left(\mathcal{S}_{1}\right)\right) \subseteq N_{\mathcal{V}}\left(\sigma\left(\mathcal{S}_{2}\right)\right)$. Assume otherwise. Let $a \in u\left(\sigma\left(\diamond\left(p_{1}, \ldots, p_{n}\right)\right)\right.$. $v\left(a, \sigma\left(\diamond\left(p_{1}, \ldots, p_{n}\right)\right)\right)=t$, and since $\mathcal{W}$ is G-legal, $r$ is not fulfilled in $a$ by $\sigma$. By 2 and 3 , either $a \notin D_{\mathcal{V}}\left(\sigma\left(\mathcal{S}_{1}\right)\right)$ or $a \in N_{\mathcal{V}}\left(\sigma\left(\mathcal{S}_{2}\right)\right)$. Let $b \in W$ such that $b \in D_{\mathcal{V}}\left(\sigma\left(\mathcal{S}_{1}\right)\right) \backslash N_{\mathcal{V}}\left(\sigma\left(\mathcal{S}_{2}\right)\right)$. Again by 2 and 3, every $s \in \sigma\left(\mathcal{S}_{1}\right)$ is true in $b$, and every $s \in \sigma\left(\mathcal{S}_{2}\right)$ is locally true in $b$. It follows that $\sigma$ fulfils $r$ in $b$, and since $\mathcal{W}$ is $\mathbf{G}$-legal, $v\left(b, \sigma\left(\diamond\left(p_{1}, \ldots, p_{n}\right)\right)\right)=f$. Using the persistence condition, $b<a$. Since $b \in D_{\mathcal{V}}\left(\sigma\left(\mathcal{S}_{1}\right)\right)$, $a \in D_{\mathcal{V}}\left(\sigma\left(\mathcal{S}_{1}\right)\right.$ ) (every element of $V$ is upwards closed). It follows that $a \in N_{\mathcal{V}}\left(\sigma\left(\mathcal{S}_{2}\right)\right)$, and so $a \in D_{\mathcal{V}}\left(\sigma\left(\mathcal{S}_{1}\right)\right) \rightarrow N_{\mathcal{V}}\left(\sigma\left(\mathcal{S}_{2}\right)\right)$.

5 . One direction easily follows from 2. The converse is easy and left to the reader.

\footnotetext{
${ }^{3}$ A subset $A$ of $W$ is upwards closed if $u \in A$ whenever $w \in A$ and $w \leq u$
}

Corollary 39 (Soundness and Completeness). Every coherent canonical Gödel system $\mathbf{G}$ is strongly sound and complete with respect to the semantics of $\mathbf{G}$-legal Gödel valuations. In other words: $\mathcal{H} \vdash_{\mathbf{G}} H$ iff $\mathcal{H} \vDash_{\mathbf{G}} H$.

Proof. Assume $\mathcal{H} \vdash_{\mathbf{G}} H$. By Theorem $34 \mathcal{H} \vDash_{\mathbf{G}}^{K} H$. Let $\mathcal{V}$ be a $\mathbf{G}$-legal $\mathcal{L}$-Gödel valuation which is a model of $\mathcal{H}$. We show that it is a model of $H$. Let $\mathcal{W}=\langle V, \geq, v\rangle$, where $v$ is defined as in Lemma 37 . By Lemma $37, \mathcal{W}$ is a $\mathbf{G}$-legal $\mathcal{L}$-Gödel frame which is a model of $\mathcal{H}$. It follows that $\mathcal{W}$ is a model of $H$, and Lemma 37 again implies that $\mathcal{V}$ is a model of $H$. The converse is analogous (using Lemma 38).

\section{Acknowledgements}

This research was supported by The Israel Science Foundation (grant no. 280-10).

\section{References}

[1] A. Avron, Using Hypersequents in Proof Systems for Non-classical Logics, Annals of Mathematics and Artificial Intelligence, 4, 225-248, 1991.

[2] A. Avron, A Simple Proof of Completeness and Cut-admissibility for Propositional Gödel Logic, Journal of Logic and Computation, doi: 10.1093/logcom/exp055, 2009.

[3] A. Avron and I. Lev, Non-deterministic Multivalued Structures, Journal of Logic and Computation, vol. 15, 241-261, 2005. A conference version: Canonical Propositional Gentzen-Type Systems, In Goré, R., Leitsch, A., Nipkow, T., (eds.) IJCAR 2001, LNCS (LNAI), vol. 2083, pp. 529-544. Springer, Heidelberg, 2001.

[4] A. Avron and O. Lahav, On Constructive Connectives and Systems, Journal of Logical Methods in Computer Science, 6, 2010.

[5] M. Baaz, A. Ciabattoni and C. G. Fermüller, Hypersequent Calculi for Gödel Logics - a Survey, Journal of Logic and Computation, 13:835861, 2003.

[6] P. Hájek, Metamathematics of Fuzzy Logic, Kluwer Academic Publishers, 1998.

[7] G. Metcalfe, N. Olivetti, D. Gabbay, Proof Theory for Fuzzy Logics, Springer, 2009.

[8] G. Sundholm, Proof theory and Meaning, in Handbook of Philosophical Logic (D. M. Gabbay and F. Guenthner, eds), 2nd ed., Vol 9, 165198, 2002. 